\title{
A COMPARISON OF CONTEMPORARY PROTOTYPING METHODS
}

\author{
Coutts, Euan Ross (1,2); Wodehouse, Andrew (2); Robertson, Jason (2) \\ 1: University of Canterbury; 2: University of Strathclyde
}

\begin{abstract}
Prototypes are a common feature of many product design and development endeavours. An ever widening range of prototyping options are available to designers and engineers. May particular options be superior to others, or more appropriate for particular endeavours? This paper reviews current literature on the nature of what constitutes a prototype and the benefits they offer to the discipline. They principally facilitate communication, aid learning, help gain and provide feedback, inform decision making and generally provide superior design outcomes. In order to determine if any particular manner of prototype is preferable for achieving these benefits a comparative study of some of the contemporary prototyping methods is subsequently conducted: A 3D printed prototype (physical prototype), a CAD prototype (represented using a computer monitor), an augmented reality prototype (represented using a tablet device) and a virtual reality prototype (represented using a stereo projector and polarised glasses). The results indicate that while all provide benefits, overall the physical prototype performs best and the augmented reality prototype performs most poorly.
\end{abstract}

Keywords: Virtual reality, 3D printing, Product modelling / models, Prototyping

\section{Contact:}

Coutts, Euan Ross

University of Canterbury, New Zealand

School of Product Design

New Zealand

euan.coutts@canterbury.ac.nz

Cite this article: Coutts, E.R., Wodehouse, A., Robertson, J. (2019) 'A Comparison of Contemporary Prototyping Methods', in Proceedings of the 22nd International Conference on Engineering Design (ICED19), Delft, The Netherlands, 5-8 August 2019. DOI:10.1017/dsi.2019.137 


\section{INTRODUCTION}

Prototypes are a common feature of many product design and development endeavours. As technology continues to evolve, the formats and types of prototype available to designers has widened considerably. In recent years, the emergence of digital technologies has presented options in additive manufacture, augmented reality and virtual reality that can accelerate and enhance design decision making. However, the nature and benefits of these are not yet well understood - how should these differing formats of prototypes be deployed, presented and utilised? This paper advances understanding of what constitutes a prototype and progresses to compare several prototyping options through a practical study. This paper advances understanding of the benefits of different prototyping formats, and evaluates communication performance and designer preferences through a comparative study.

Terminology in this domain - prototype, model, mock-up etc. - are often used interchangeably. The Academic Press Dictionary of Science and Technology gives the definition of prototype as "an early or original form... (in engineering) a full-scale model of a structure or piece of equipment used in evaluating form, design, fit, and performance" (Morris, 2018). Similarly, the definition given for a mockup is given as "a scale model, often full size, of a structure, apparatus or vehicle; used for study, training, or testing and to determine if the apparatus can be manufactured easily and economically." Both definitions describe a scale model that is used for analysis and testing, this leads to the conclusion that both terms can be used to describe the same thing. This also falls into line with both literature (Wang, 2002) and real world practises that suggests they are commonly used as interchangeable terms.

Lauff, Kotys-Schwartz and Rentschler (2018) ask the question "what is a prototype?" within the context of the design process, stating "A prototype is a physical or digital embodiment of critical elements of the intended design, and an iterative tool to enhance communication, enable learning, and inform decisionmaking at any point in the design process". This definition was derived through ethnographic observations and inductive analysis of three design companies and states three key aspects:

- That a prototype is an embodiment of a design.

- It is a tool to improve communication, learning and decision making.

- It can be used at any stage in the design process.

However, Houde and Hill (1997) raise the observation that the term "prototype" is ambiguous, meaning different things to different people, for example a designer may call a moulded foam object a prototype and a programmer would call a test program a prototype. Additionally, an example asking "is a brick a prototype?" is given, the answer to which "depends on what it is used for, if it is used to represent the weight and scale of a future artefact then it must be a prototype". This illustrates that a prototype does not rely on how it is manifest, what defines a prototype is that it is used to explore or demonstrate some aspect of a future artefact, by such logic any representation of a design may arguably be considered a prototype. This illustrates that the fundamental function of a prototype is to communicate design intent, and to this extent any suitable representation of a design may arguably considered a prototype. Indeed, other research has included sketches and illustrations within the working definition. This research, however, focuses on 3D representations that provide some form of interaction in the demonstration and communication of a design concept.

In this vein, a prototype is often considered as a means to answer a question, such as proving an application of a technology, or clearly communicating some key functionality of a design, frequently referred to as "proof of concept" (Ullman, 2010). This work's definition of a prototype is aligned with Lauff et al. (2018), Lidwell et al. (2003) and galvanised by Houde and Hill's earlier definition. It is asserted that a prototype is an external artefact which embodies certain, or all, features of an intended design. Such artefacts are external to the designer (does not exist only in designers mind) and may take many forms and be created by a variety of prototyping methods.

\section{PROTOTYPES AND PROTOTYPING METHODS}

Prototypes can be enormously useful within the design process, having many purposes and uses. Multiple papers and books propose different forms of prototyping each with their own benefits and methods. Ulrich and Eppinger, (2012) describe the purpose of a prototype as being able to aid learning, communication, integration and milestones. This categorisation of prototypes allows designers to establish, from the beginning of a prototyping exercise, what the prototype they are about to create is for. This has the benefit of allowing the prototype to be created with a fixed purpose in mind, helping to 
ensure that it meets that purpose, and may additionally reduce the possibility of hardware swamping (Clausing, 1998). Ullman, (2010) takes a different point of view, by describing different types of prototypes in terms of their purpose and at what phase in a product development they should be used, describing proof of concept, proof of product, proof of process and proof of production. While Ullman does provide more specifics and guidelines on what each prototype is for and how it should be manifest, his list is somewhat constrained in terms of scope, there is no mention of a prototype used to communicate. An additional observation would be that each type is mainly focused on validation and verification.

Prototyping not only refers to the creation of prototypes, it also implies that prototypes are created and used for a specific purpose (Pidaparti, 2018; Ulrich and Eppinger, 2012). It can be viewed as a step, task or process within the design process, where the output is ultimately evaluated, with this evaluation feeding back into the design process, seeding the development or validation of further concepts. The way in which someone conducts a prototyping process will have some effect on the outputs of the process, not just in what prototypes are created but what is gained from the activity. Structures for prototyping processes have been proposed in some papers and even tested for validity (Christie, et al., 2012; Camburn, et al., 2013a). While purposes and intent can vary across design contexts, for any model that is shared across the design team and with key stakeholders, the accuracy and resolution of representation is critical to its effectiveness. In this regard, the research here assumes that the purpose of the prototype is to communicate design ideas from designer to designer, or from designer to client, in order to support discussions and decision making as part of the design process. Prototypes constructed for the purposes of individual exploration, or 'internal conversation', as discussed by Goldschmidt (2006) are not considered at this time.

Prototypes can come in a near infinite number of shapes and sizes, however they can be generally be placed on a spectrum of high-fidelity (hi-fi) to low-fidelity (lo-fi), the fidelity of a prototype refers to how closely it resembles or how similar it is to the desired product (Yang, 2005; Houde, S. and Hill, C., 1997). Lo-fi prototyping it characterised by quick and easy creation of an artefact. Lo-fi is also known as low-tech as the means to create them consists mostly of time, with some cardboard, sticky tape, post-it notesor similar. This type of prototyping has the benefit of being low cost and that it allows non- professionals to actively participate in the design process (Egger, 2000).

At the opposite end of the spectrum there is hi-fi prototyping, in which a prototype is often created using the same materials and production methods as the final product, intended to have the same functionality and appearance as a final product (Walker, Takayama and Landay, 2002; Yang, 2005). Hi-fi prototypes take more time, money and resources to make that lo-fi prototypes (Egger, 2000). The question of which is best used depends highly on what they are being used for and is more than the simple question of how much time and money is available. Several studies have investigated this question, Walker, Takayama and Landay (2002), found few differences between the two when testing web prototypes, as the feedback gained from both was equally as good. This is supported by the findings of Sauer, Seibel and Rüttinger (2010), who also found that users are capable of extrapolating from lo-fi prototypes, meaning they could infer functionality and problems that were not there. Both types of prototypes have their place within the design process, with each being useful for different things (Rudd, Stern and Isensee, 1996).

\section{EXPERIMENTAL DESIGN}

An experiment was designed to further investigate how different prototyping methods may affect communication of a design's properties. Four prototyping methods were used to create proof-of-concept prototypes (Ullman, 2010), and more specifically learning prototypes (Ulrich and Eppinger, 2012). They were evaluated on how well they act as integration prototypes within Houde and Hill's (1997) model. This evaluation therefore has three categories: "role", "look and feel", and "implementation". While Houde and Hill's work may initially appear a little dated, the principles for determining the efficacy of a prototype are robust. However, the means of prototyping have moved on considerably since the 90's; with the advent and increasing affordability of virtual and augmented reality platforms for prototyping how do these contemporary methods of prototyping compare in achieving the purpose of a prototype?

Additive manufacturing (3D printing), augmented reality, virtual reality and Computer Aided Design (CAD) represent the primary emerging digital prototyping methods available to designers. All of these, at their core, require some form of digital model that they represent. Using an identical model for each method, allows for a comparison of the prototyping methods; the same artefact (be it digital 
or physical) is being displayed in each method, meaning that any differences observed are a result of only the prototyping methods used. To prevent bias due to variation of the artefact, a physical prototyping method that can utilise the same digital modal used by the virtual methods is key. For this purpose, two possible methods were identified, these being CNC machining and 3D printing. While both were considered the difference would only be materials used, rather than differences in the methods themselves. As a result, 3D printing was included as the sole physical prototyping method.

The artefact chosen to be prototyped in the study would require some form of mechanism and yet would need to be sufficiently familiar, or ubiquitous, to the participants. To fulfil these requirements a CAD assembly of a cork screw was selected, as it is relatively simple while still having a mechanism that required a degree of interrogation in order to review configuration and functionality, while at the same time being a familiar object. The part file was obtained from Grab CAD (Crasto, 2018).

Figure 1 shows the different incarnations of the corkscrew product used in the study:

(a) Virtual reality; displayed using a stereo projector and polarised glasses.

(b) Augmented reality displayed on an iPad that was running the e drawings app.

(c) CAD displayed on a laptop.

(d) $3 \mathrm{D}$ printed prototype created in blue $\mathrm{ABD}$ plastic at a $0.2 \mathrm{~mm}$ layer height.
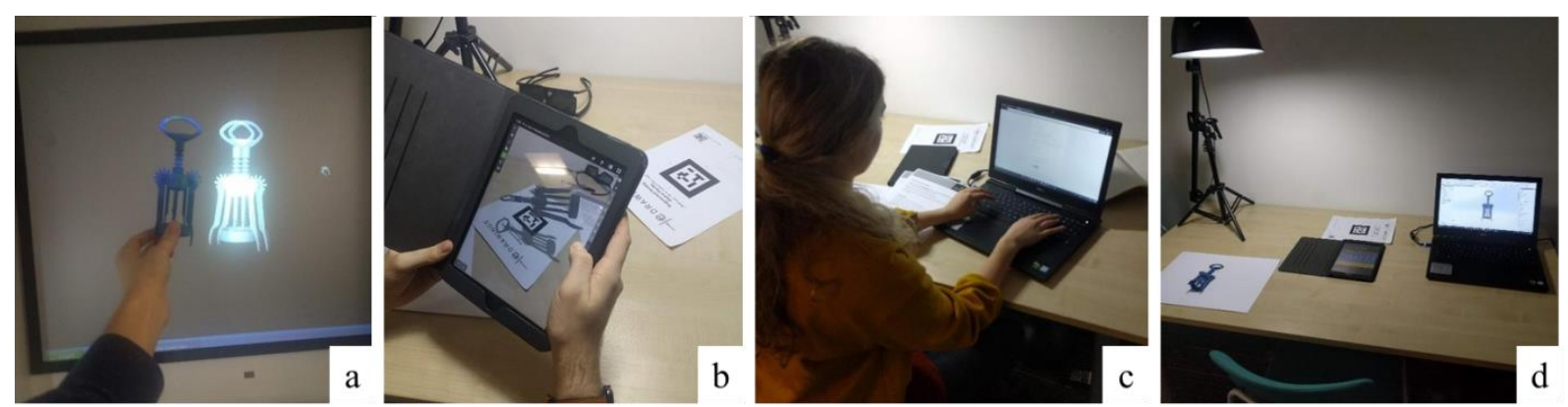

Figure 1. Different prototyping methods used in the study

\subsection{Evaluation}

A survey was constructed consisting of 3 sections, the first section contained two screening questions, used to ensure participants were designers familiar with prototypes, preventing those who do not belong to the research population being included (Brace, 2004). A total of 30 trainee design engineers, with a minimum of three years training, participated in the study. The main body of the questionnaire consisted of three questions that were repeated for each of the four prototypes. These questions asked:

- How well could you determine the final product's functionality, what it's function or job is?

- How well could you determine the look, feel and sound of the final product?

- How difficult was it to understand how it operates?

These questions were answered on a semantic differential scale, with the dimensions being composed of 6 points, that were rated from "with great difficulty" to "very easily" for the first two questions and "very difficult" to "very easy" for the third. The participants were additionally prompted to answer an open question for any additional information.

The last part of the survey was more general, it asked:

- Which of the prototyping methods did you like the most?

- Which of the prototyping methods did you find the most useful?

These questions were also followed up by an additional probing question, asking "could you please explain your answer". This last section was included as it is more open, and opinion based, than the first two. This provided participants a chance to answer the questions however they liked, this allowed for their opinions on what makes a "good" prototyping method.

\section{RESULTS}

The results below show the total mean scores for each prototyping method. These were generated by taking the mean of each aspect that was rated (roll, look \& feel and implementation) and then taking the mean of those 3 , the result is the average score the participants gave each prototyping method. 


\subsection{Rating}

Figure 2 shows that the physical prototype, on average, was the best of the four at communicating all aspects, while the AR prototype was rated the poorest, on average. Figure 3 compares the percentage of the total ratings that were positive and negative for each prototyping method. This perspective on the results shows that overall there were more positive ratings for the virtual reality prototype than the CAD prototype, helping to reinforce the mean score findings. Overall the AR prototype is the only one that has more negative votes than positive.

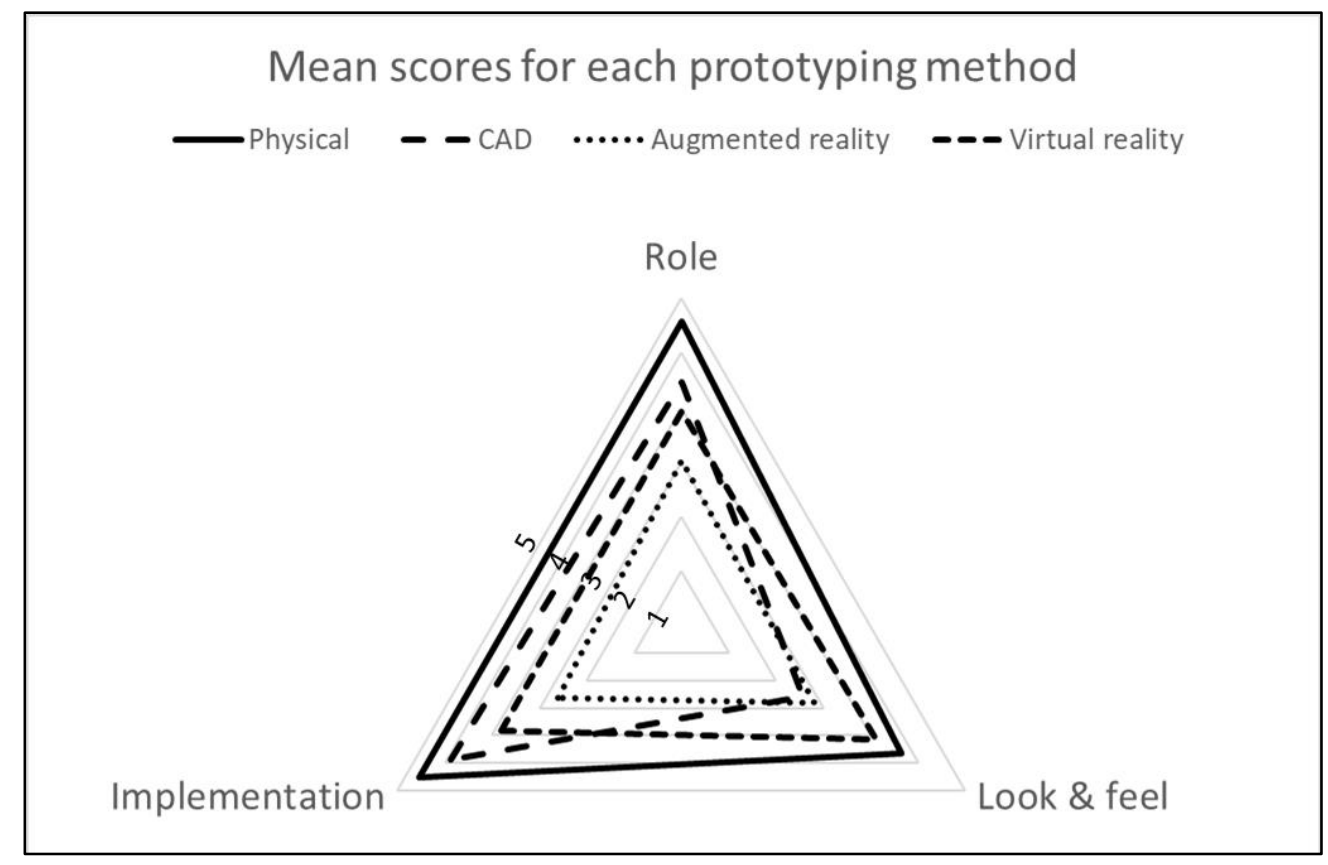

Figure 2. Mean scores for each prototyping method.

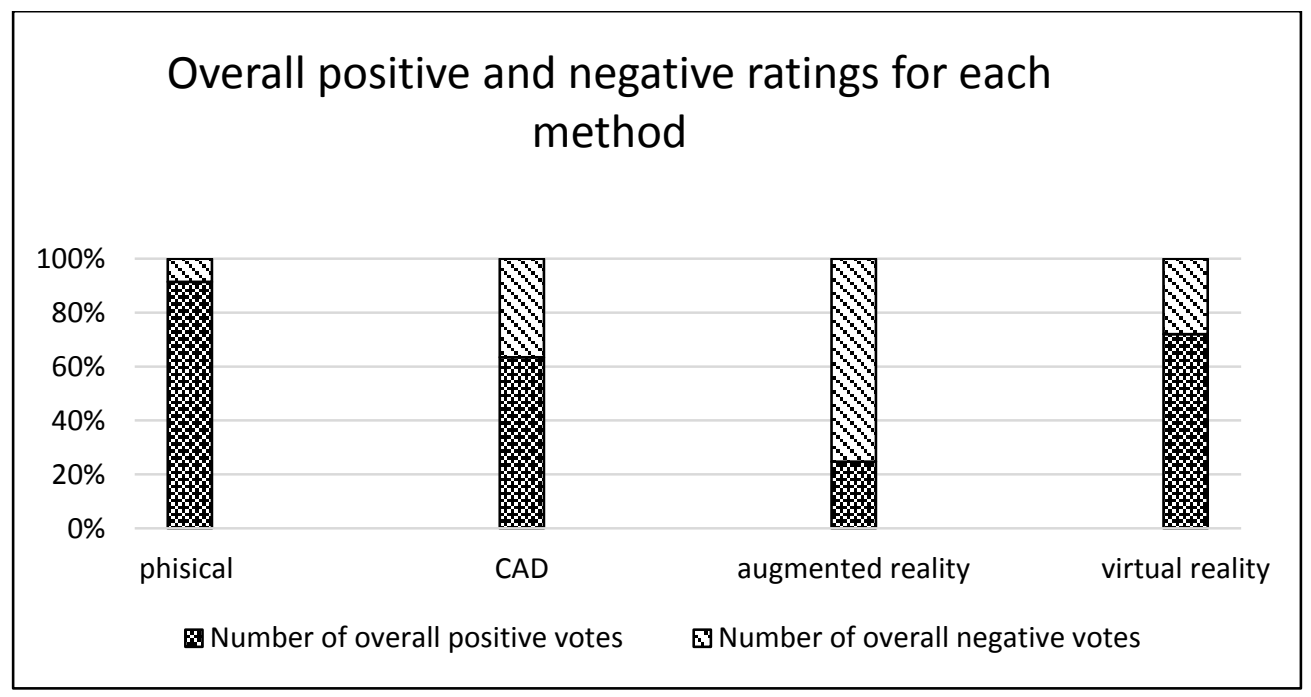

Figure 3. Overall positive and negative ratings for each prototyping method.

\subsection{Preference}

Figure 4 details what method the participants identified as their preferred method, they did not need to provide a reason, although some did, and this question was based entirely on their own opinion. More than $50 \%$ of the participants in the investigation voted the AR prototype as their favourite, which was twice that of second place. Most of the reasons stated for AR prototype preference were that it was "cool", "new", "interesting" and "techy". While the reasons given for selecting the physical prototype were entirely based around it being functional and the participant could hold it in their hands. Participants were then asked what method they found to be the most useful, as shown in Figure 5. 
Most participants thought that the physical prototype was the most useful, with most reasons given mentioning that it is the only one that allows the prototype to be held in the hand and felt.

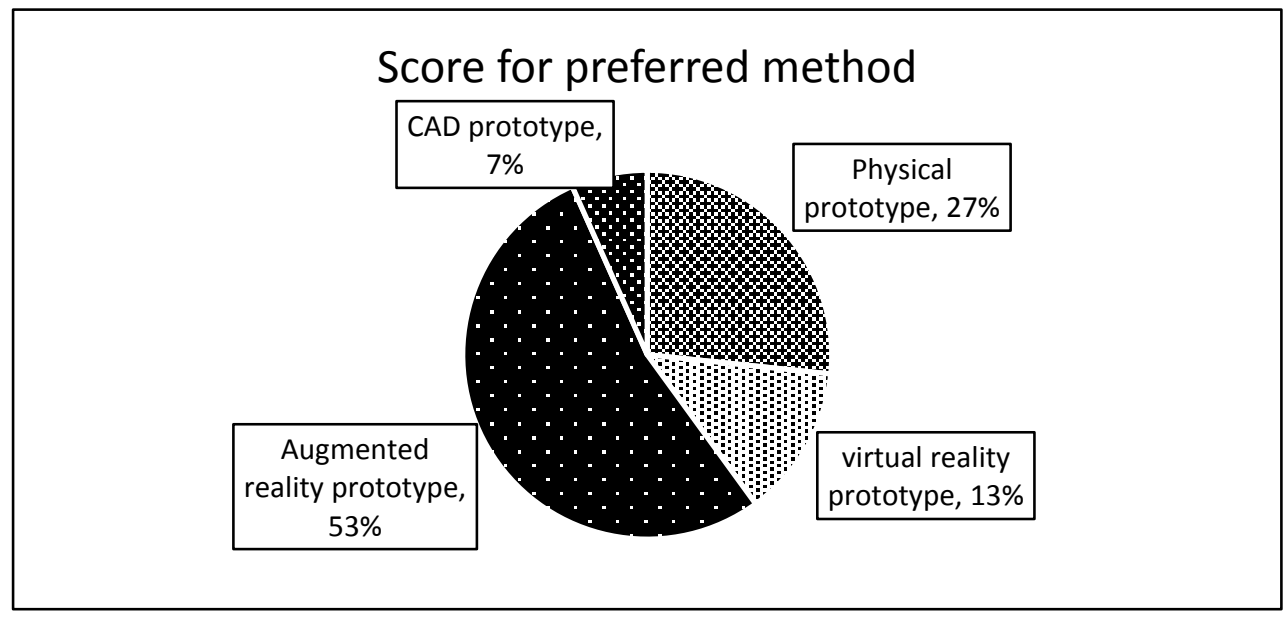

Figure 4. Score for preferred method.

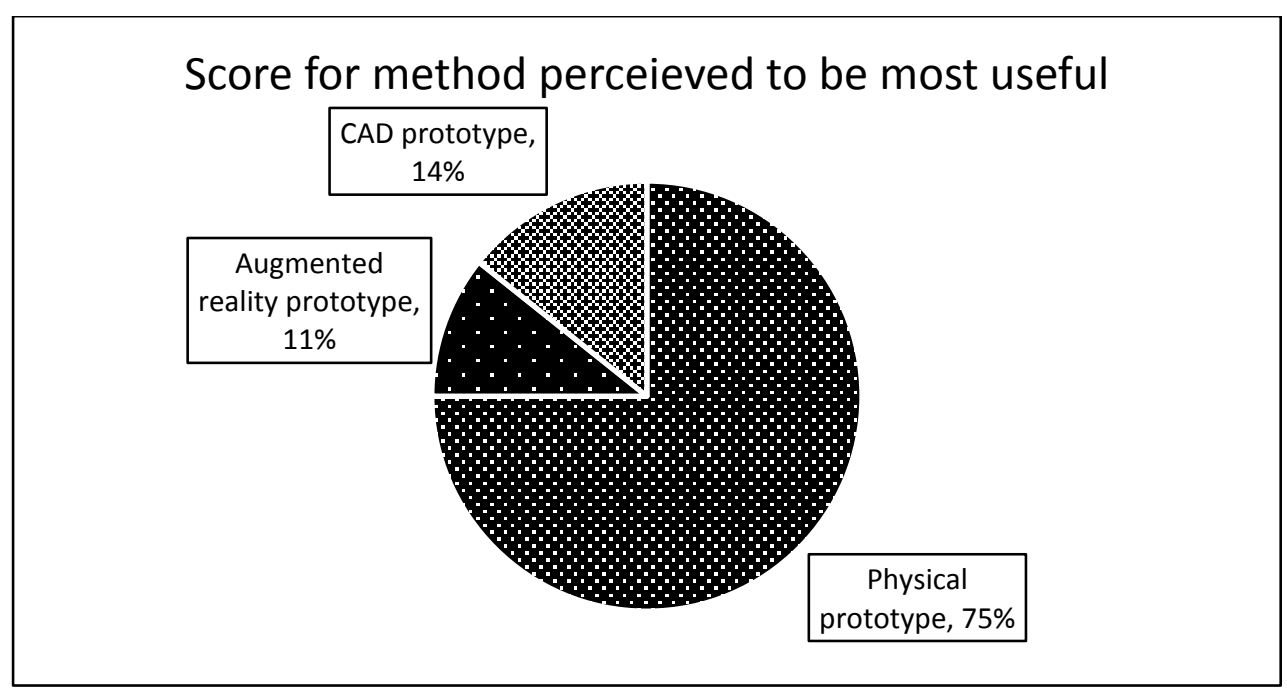

Figure 5. Score for method perceived to be most useful.

\section{DISCUSSION \& CONCLUSION}

Prototypes and prototyping in general have existed for many years and presumably will do for years to come, for this to be the case there are benefits to these artefacts and processes. While individual prototypes can differ vastly from each other, prototyping when considered as a process or technique is far more clearer in terms of what it can offer individuals and teams. Many authors have asked the question "What do you gain from prototyping?" often only looking at specific areas, although some have tried to cover the entire subject. There is some consensus between authors on a few of the general answers, although many approaches each differently.

Prototyping helps communicate ideas (Buchenau and Suri, 2000). This is achieved by synchronising the mental model individuals have of a concept, ether passively acting as a representation or actively by providing a mechanism for discussion, these faculties in turn allow the concept to be explained, allow others to provide focused feedback on the specifics of a design and these two combined allow for meaningful negotiations to be conducted about the prototype, product or idea, (Lauff et al., 2018). This communication between team members does not only apply to designers it may apply to managers, clients and other stakeholders (Gerber and Carroll, 2012). Part of this learning process is feedback, prototypes provide feedback in many ways, such as showing how components interact and fit together. Within design and the design of a new product feedback is essential, and arguably the most important feedback is gained from the intended end user. Prototypes can act as excellent tools to gain user 
feedback, (Steven Dow et al., 2009; Lui and Khooshabeh, 2003), as they allow the user to share the identical mental image of a product and provide something that they can test and interact with.

Such feedback can represent a paradox; it is both essential and innately useless. Regardless of how much feedback is gained if it is not acted upon to make decisions, it is only data. Decision making is fundamentally important to the design process, not only for improvements to be implemented but to move between key stages in the design process and ensuring the deadlines are met, (Yilmaz et al., 2013). Prototypes have been shown to be useful for decision making where there are various competing recruitments involved (Doke and Swanson, 1995; Smith and Eppinger, 1997). While feedback it still the heart of good decision making prototyping and prototypes can provide this feedback, (Krishnan and Ulrich, 2001). Prototypes inform decision making, as they provide a combined ability to aid communication (including gaining feedback) and facilitate learning (Lauff et al., 2018).

Informed decision making can be the making or breaking of a project, this also applies to the decision of when and how prototyping could be used within a project. Implementing it early has been shown to increase the quality of a final product (Yang, 2005). This increase in quality through prototyping is not limited to only the earlier stages however, an increase in technical quality can be seen though the implementation of prototyping within a project (Steven Dow et al., 2009). One of the faculties of prototyping that can provide this improvement of results is its ability to allow individuals and teams to explore parallel design concepts (Steven Dow et al., 2011; Walker, Takayama and Landay, 2002).

From literature there appears to be a lot to be gained from prototyping, and by extension prototypes. Generalising and combining the finding, prototypes and prototyping provide five benefits, which are that they:

- facilitate communication

- $\quad$ aid learning

- $\quad$ help gain and provide feedback

- $\quad$ inform decision making

- $\quad$ provide superior design outcomes

The results of the investigation indicate that physical prototyping should be employed if the purpose of the prototype is to communicate the role and implementation of a product. However, if the scale and cost of a physical prototype become prohibitive, CAD prototypes should be considered as this method is regarded as similar to that of the physical but with the added benefit of not affected as much by cost and size constraints.

The apparent favouring of physical prototyping methods over virtual based prototyping methods appears to be a result of participants being able to hold and feel the prototype. This tactile feedback is lacking in other methods, although the question of scale arises again, as this may not be the case for large products that do not fit into the hand. Further study could investigate how the results of an investigation like this one change when multiple products of various sizes are considered.

Virtual reality only excelled in one area, describing the look $\&$ feel of a product, this is assumed to be a result of it being 3D, which may have allowed it to describe the form and shape of the corkscrew well. On the other hand, its performance was comparable to that of the physical prototype. This ultimately leads back to the questions of scale and cost however, if it is more economical and practical to use a virtual reality prototype then it should be considered, but only if it is for communicating look and feel, if there are other considerations than physical may be worth the investment.

The AR prototype performed the worst in all the aspects considered but it was rated by a large majority to be there favourite method. The reasons given all related to the method being "cool" or "new", which strongly suggest this is a result of "the wonder of the new". However, this is speculative at this stage although the results suggest this it could be a potentially fruitful area of investigation. The results would appear to suggest that physical prototyping performed best overall. However, there are caveats which prevent it from being recommended in every purpose.

\section{CONCLUSION}

It is concluded from review of literature that for prototyping to be successful there must be purpose behind each prototype and the process must have an end goal in mind. The results of the experimental study would appear to indicate that physical prototyping should be employed if the purpose of the prototype is to communicate the role and implementation of a product. Which would appear to support widely held assumptions that the construction of physical models is important and effective in 
communicating (and hence exploring) the functional and mechanical aspects of a design concept, the prototype as a stimulus for discussion, as it were. However, results would also appear to indicate that augmented reality models brought the engagement, but did not necessarily deliver on the information, given subjective participants responses in explaining this preference there may be a "wonder of the new" involved in the use of these methods, although this would require further investigation. In practice, and in certain settings, this may be enough in itself and there will likely be future opportunities to make more sophisticated AR models with aspects of physical feedback. Areas for future study would be to ascertain prototypes created with different purposes and mind and how different prototyping methods may facilitate these purposes.

\section{REFERENCES}

Brace, I. (2004), Questionnaire Design : How to Plan, Structure and Write Survey Material for Effective Market Research. 2nd ed. Kogan Page, Limited, London, pp. 63-126.

Dunlap, B.U., Hamon, C.L., Camburn, B.A., Crawford, R.H., Jensen, D.D., Green, M.G., Otto, K. and Wood, K.L. (2014), "Heuristics-based prototyping strategy formation: Development and testing of a new prototyping planning tool", (2014), In: ASME 2014 International Mechanical Engineering Congress and Exposition. ASME 2014, p. 9.

Buchenau, M. and Suri, J.F. (2000), Experience Prototyping Proceedings of the Conference on Designing Interactive Systems Processes Practices Methods and Techniques, pp. 424-433.

Camburn, Dunlap, B.U., Kuhr, R., Viswanathan, V.K., Linsey, J.S., Jensen, D.D., B., Dunlap, B., Kuhr, R., Viswanathan, V., Linsey, J. and Jensen, D. et al. (2013a), "Methods for prototyping strategies in conceptual phases of Design: Framework and experimental assessment," In: ASME 2013 Power Transmission and Gearing Conference. Portland: Volume 5: 25th International Conference on Design Theory and Methodology, p. 10.

Camburn, B.A., Dunlap, B.U., Linsey, J., Viswanathan, V., Jensen, D., Crawford, R., Otto, K. and Wood, K.L. (2013b), "Using design problem characteristics to build a prototyping strategy," ASEE Annual Conference, Atlanta, GA.

Crasto, M.C. (2018), Grab CAD. [Online]. [3 December 2018]. Available from: https://grabcad.com/library/cork-screw-3

Christie, E.J., Jensen, D.D., Buckley, R.T., Menefee, D.A., Ziegler, K.K. and Wood, P.K.L. et al. (2012), "Prototyping strategies: Literature review and identification of critical variables", Proceedings of the 2012 American Society for Engineering Education Conference

Clausing, D. (1998), Total Quality Development, 2nd ed, ASME Press, New York.

Doke, E. and Swanson, N. (1995), "Decision variables for selecting prototyping in information systems development: A Delphi study of MIS managers", Information \& Management, Vol. 29 No. 4, pp. 173-182.

Dow, S.P., Glassco, A., Kass, J., Schwarz, M. and Klemmer, S.R. (2009), "The effect of parallel prototyping on design performance, learning, and self-efficacy", Stanford Tech Report. [online] Stanford: Stanford University HCI Group. Available at: https://hci.stanford.edu/publications/2009/EffectOfParallelPrototyping.pdf [Accessed 1 Dec. 2018].

Dow, S., Fortuna, J., Schwartz, D., Altringer, B., Schwartz, D. and Klemmer, S. (2011), "Prototyping dynamics: sharing multiple designs improves exploration, group rapport, and results", In: Conference on Human Factors in Computing Systems, AMC, New York, NY, USA, pp. 2807-2816.

Egger, F.N. (2000), "Lo-Fi vs. Hi-Fi Prototyping: how real does the real thing have to be?", Teaching HCI Workshop, OZCHI2000, Sydney (Australia), December 14-19, 2000

Gerber, E. and Carroll, M. (2012), "The psychological experience of prototyping," Design Studies, Vol. 33 No. 1, pp. 64-84.

Goldschmidt, G. and Smolkov, M. (2006), "Variances in the impact of visual stimuli on design problem solving performance," Des. Stud., Vol. 27 No. 5, pp. 549-569.

Houde, S. and Hill, C. (1997), "What Do Prototypes Prototype", Handbook of Human-Computer Interaction, 2nd ed, Elsevier Science, Amsterdam, pp. 367-381.

Krishnan, V. and Ulrich, K. (2001), "Product development decisions: A review of the literature", Management Science, Vol. 47 No. 1, pp. 1-21.

Lauff, C., Kotys-Schwartz, D. and Rentschler, M. (2018), "What is a prototype? What are the roles of prototypes in companies?", Journal of Mechanical Design, Vol. 140 No. 6, p. 061102.

Lidwell, W., Holden, K. and Butler, J. (2003), Universal Principles of Design, Rockport Publishers, Gloucester, MA.

Lui, L. and Khooshabeh, P. (2003), "Paper or interactive?: a study of prototyping techniques for ubiquitous computing environments", In: Extended Abstracts on Human Factors in Computing Systems, AMC, New York, NY, USA, pp. 1030-1031. 
Morris, C. (2018), "Prototype, engineering”, In: Academic Press Dictionary of Science and Technology, 1 st ed. [online] Academic Press, San Diego, p. 1744.

Rudd, J., Stern, K. and Isensee, S. (1996), "Low vs. high-fidelity prototyping debate”, Interactions, Vol. 3 No. 1 , pp. 76-85.

Sauer, J., Seibel, K. and Rüttinger, B. (2010), "The influence of user expertise and prototype fidelity in usability tests”, Applied Ergonomics, Vol. 41 No. 1, pp. 130-140.

Smith, R. and Eppinger, S. (1997), “A Predictive Model of Sequential Iteration in Engineering Design”, Management Science, Vol. 43 No. 8, pp. 1104-1120

Ullman, D. (2010), The Mechanical Design Process, 4th ed, McGraw-Hill, Boston, pp. 117-131.

Ulrich, K. and Eppinger, S. (2012), Product Design and Development, 5th ed, Irwin/McGraw-Hill, Boston, Mass., pp. 289-309.

Wall, M., Ulrich, K. and Flowers, W. (1992), "Evaluating prototyping technologies for product design", Research in Engineering Design, Vol. 3 No. 3, pp. 163-177.

Wang, G. (2002), "Definition and review of virtual prototyping", Journal of Computing and Information Science in Engineering, Vol. 2 No. 3, pp. 232-236.

Walker, M., Takayama, L. and Landay, J. (2002), "High-fidelity or low-fidelity, paper or computer? Choosing attributes when testing web prototypes", Proceedings of the Human Factors and Ergonomics Society Annual Meeting, Vol. 46 No. 5, pp. 661-665.

Yang, M. (2005), “A study of prototypes, design activity, and design outcome”, Design Studies, Vol. 26 No. 6 , pp. 649-669.

Yilmaz, S., Daly, S., Christian, J., Seifert, C. and Gonzalez, R. (2013), “Can experienced designers learn from new tools? A case study of idea generation in a professional engineering team", International Journal of Design Creativity and Innovation, Vol. 2 No. 2, pp. 82-96. 
